\title{
Combined linkage and association mapping reveals candidates for $S c m v 1$, a major locus involved in resistance to sugarcane mosaic virus (SCMV) in maize
}

Yongfu Tao ${ }^{1 \dagger}$, Lu Jiang ${ }^{1 \dagger}$, Qingqing Liu ${ }^{1 \dagger}$, Yan Zhang ${ }^{1}$, Rui Zhang ${ }^{1}$, Christina Roenn Ingvardsen ${ }^{3}$, Ursula Karoline Frei ${ }^{2}$, Baobao Wang ${ }^{1}$, Jinsheng Lai ${ }^{1}$, Thomas Lübberstedt ${ }^{2}$ and Mingliang $\mathrm{Xu}^{1 *}$

\begin{abstract}
Background: Sugarcane mosaic virus (SCMV) disease causes substantial losses of grain yield and forage biomass in susceptible maize cultivars. Maize resistance to SCMV is associated with two dominant genes, Scmv1 and Scmv2, which are located on the short arm of chromosome 6 and near the centromere region of chromosome 3, respectively. We combined both linkage and association mapping to identify positional candidate genes for Scmv1.

Results: Scmv1 was fine-mapped in a segregating population derived from near-isogenic lines and further validated and fine-mapped using two recombinant inbred line populations. The combined results assigned the $5 \mathrm{cmv} 1$ locus to a 59.21-kb interval, and candidate genes within this region were predicted based on the publicly available B73 sequence. None of three predicted genes that are possibly involved in the disease resistance response are similar to receptor-like resistance genes. Candidate gene-based association mapping was conducted using a panel of 94 inbred lines with variable resistance to SCMV. A presence/absence variation (PAV) in the Scmv1 region and two polymorphic sites around the Zmtrx-h gene were significantly associated with SCMV resistance.

Conclusion: Combined linkage and association mapping pinpoints $Z m t r x-h$ as the most likely positional candidate gene for Scmv1. These results pave the way towards cloning of Scmv 1 and facilitate marker-assisted selection for potyvirus resistance in maize.
\end{abstract}

Keywords: Maize, SCMV, QTL, Fine-mapping, Association mapping

\section{Background}

Sugarcane mosaic virus is one of the most prevalent viral pathogens, causing significant global losses in maize grain and forage yields [1], especially in China and Germany [1,2]. A recent report of SCMV in Poland and Central France revealed that the incidence of SCMV continues to increase $[3,4]$. The diagnostic symptoms of infection with SCMV include stunting, chlorosis, and reduction in plant weight and grain yield $[1,5]$. SCMV, formerly denoted as maize dwarf mosaic virus (MDMV) strain $\mathrm{B}$, and belongs to the family Potyviridae [5]. Other

\footnotetext{
* Correspondence: mxu@cau.edu.cn

${ }^{+}$Equal contributors

${ }^{1}$ National Maize Improvement Center, China Agricultural University, 2 West

Yuanmingyuan Road, Beijing 100193, People's Republic of China

Full list of author information is available at the end of the article
}

members of the family Potyviridae that also infect maize include MDMV, wheat streak mosaic virus (WSMV), Johnson grass mosaic virus (JGMV), sorghum mosaic virus (SrMV), and Zea mosaic virus (ZeMV) [6,7]. Control of SCMV by agronomic and chemical means is ineffective owing to the non-persistent transmission mode of the virus by aphid vectors. Thus, deployment of resistant varieties is the most important way to prevent yield losses, which is based on screening and identification of resistant germplasm [8].

U.S. inbred line Pa405 shows complete resistance to MDMV and SCMV under both field and greenhouse conditions after artificial inoculation [9]. In Europe, a collection of 122 early maturing maize inbred lines was screened for resistance against SCMV, resulting in the

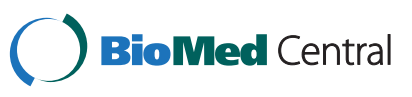


identification of three lines, D21, D32, and FAP1360A, that displayed complete resistance to SCMV [6]. In China, many elite inbred lines display complete resistance when challenged with SCMV inoculum [2,10]. Analysis of quantitative trait loci (QTL) in a cross between the resistant line D32 and susceptible line D145 revealed two major dominant genes on chromosomes 3 and 6 , and three minor QTL on chromosomes 1, 5, and 10 [11,12]. Highresolution mapping using progeny from the cross between FAP1360A (completely resistant to SCMV) and F7 (highly susceptible to SCMV) confirmed that Scmv1 (on the short arm of chromosome 6) and Scmv2 (near the centromere of chromosome 3) are crucial for maize resistance to SCMV [13]. Similar mapping results have been reported in several independent experiments $[8,14,15]$. The Scmv1 locus has also been detected in a resistant Chinese line, Huangzao4, together with additional QTL on chromosomes 1, 3, 5, and 10 [14]. In the progeny of Siyi $\times$ Mo17, two major genes were mapped on chromosome 3 (bin 3.04/05) and chromosome 6 (bin 6.00/01), respectively [8]. By using an $\mathrm{F}_{9}$-derived immortalized RIL population obtained from the cross of Huangzao $4 \times \mathrm{Mo} 17$, a major QTL was detected between chromosome bins 6.00 and 6.01 , accounting for $50.0 \%$ of the total phenotypic variation and decreasing the disease index by $27.9 \%$ [16]. The loci on chromosomes 3 and 6 have also been confirmed in tropical maize germplasm from Brazil [15]. With the aid of the whole-genome B73 sequence, the $S c m v 2$ region was fine-mapped to a region of $0.28 \mathrm{cM}$, covering a physical distance of $1,342.6 \mathrm{~kb}$. Four predicted genes possibly involved in virus movement are likely candidates for Scmv2 [17]. Existence of two closely linked resistance genes within the $S c m v 1$ region has been postulated $[18,19]$. Interestingly, several QTL that confer resistance to various viruses all map to the same region on chromosome 6 [20]. It is currently uncertain, whether a single pleitropic $S c m v 1$ gene confers resistance to different viruses, or distinct resistance genes are clustered in this region.

Even though diverse mapping populations have been used, all studies consistently identified the Scmv1 and Scmv2 genomic regions as being critical to confer resistance to SCMV. In this study, we first conducted the fine-mapping of Scmv1 using a large isogenic mapping population previously shown to segregate solely for Scmv1 [13,18,19,21,22]. Second, we employed two RIL populations from elite parental lines with contrasting resistance to SCMV to confirm and further fine map the Scmv1 locus. Third, we identified a limited number of positional Scmv1 candidate genes based on the combined fine-mapping results. Finally, we conducted candidate gene-based association mapping to characterize the QTL region and candidate genes using a panel of 94 maize inbred lines with variable resistance to SCMV.

\section{Results}

Fine-mapping of Scmv1 in a near-isogenic $F_{2}$ population Of 177 unselected $F_{2}$ plants, 50 exhibited typical symptoms of SCMV infection within 2-3 weeks after the first inoculation. The numbers of resistant and susceptible plants segregated in a 3:1 ratio $\left(\chi^{2}=0.83<\chi_{0.01,1}^{2}\right)$, in agreement with inheritance of a single dominant gene. Using the 17 polymorphic simple sequence repeat (SSR) markers available from the Maize Genetics and Genomics Database (http://www.maizegdb.org/) (Additional file 1), the Scmv1 locus was mapped between umc1018 and umc1753, with a physical distance of $18.80 \mathrm{Mb}$ according to the B73 physical map (Figure 1A). Owing to the lack of high-density markers, 28 recombinants between these two markers could not be resolved. Accordingly, we developed 11 new markers (Table 1) based on the public maize Bacterial Artificial Chromosome (BAC) sequences accessible in GenBank at the National Center for Biotechnology Information (http://www.ncbi.nlm.nih.gov). We used these 11 new markers to resolve the 28 recombinants. Subsequent marker-phenotype analysis enabled us to progressively refine the $S c m v 1$ region into a $710.97-\mathrm{kb}$ interval between STS-5 and STS-15 (Figure 1B), with still two recombinants in between those two markers. Thereafter, the markers STS-5 and STS-15 were used to genotype $510 \mathrm{~F}_{2}$ susceptible plants, resulting in 13 new recombinants. Within the STS-5/STS-15 interval, single/low copy sequences were exploited to develop seven additional markers (Table 1). These markers were used to genotype 15 recombinants, which allowed us to delimit the Scmv1 locus into a 112.39-kb interval flanked by the two closest markers, R1-2 and STS-11 (Figures 1C and 1D). There were still six recombinants to the left and eight recombinants to the right side of the Scmv1 locus (Figure 1D). Comparison with the B73 physical map indicated that the two closest markers, R1-2 and the STS-11, reside on the adjacent BACs in GenBank: b0129G15 and b0598N23, respectively.

\section{Validation of Scmv1 in RILs}

Given that the Scmv1 region could not be narrowed further using the near-isogenic $\mathrm{F}_{2}$ population, we used two RIL populations with parental lines contrasting in resistance to SCMV to confirm and fine-map the Scmv1 region. As both of the Scmv1 and Scmv2 loci are involved in maize resistance to SCMV, each RIL was investigated for its genotype at the Scmv2 locus using the co-segregating marker DJF004 developed by Ingvardsen et al. [17]. Given that one of the Scmv1 flanking markers, STS-11, failed to reveal any polymorphism between parents of each of the two RIL populations, we searched for an alternative marker around STS-11 and finally developed a new marker, B4, which is located approximately $103.75-\mathrm{kb}$ downstream of STS11. The B4 


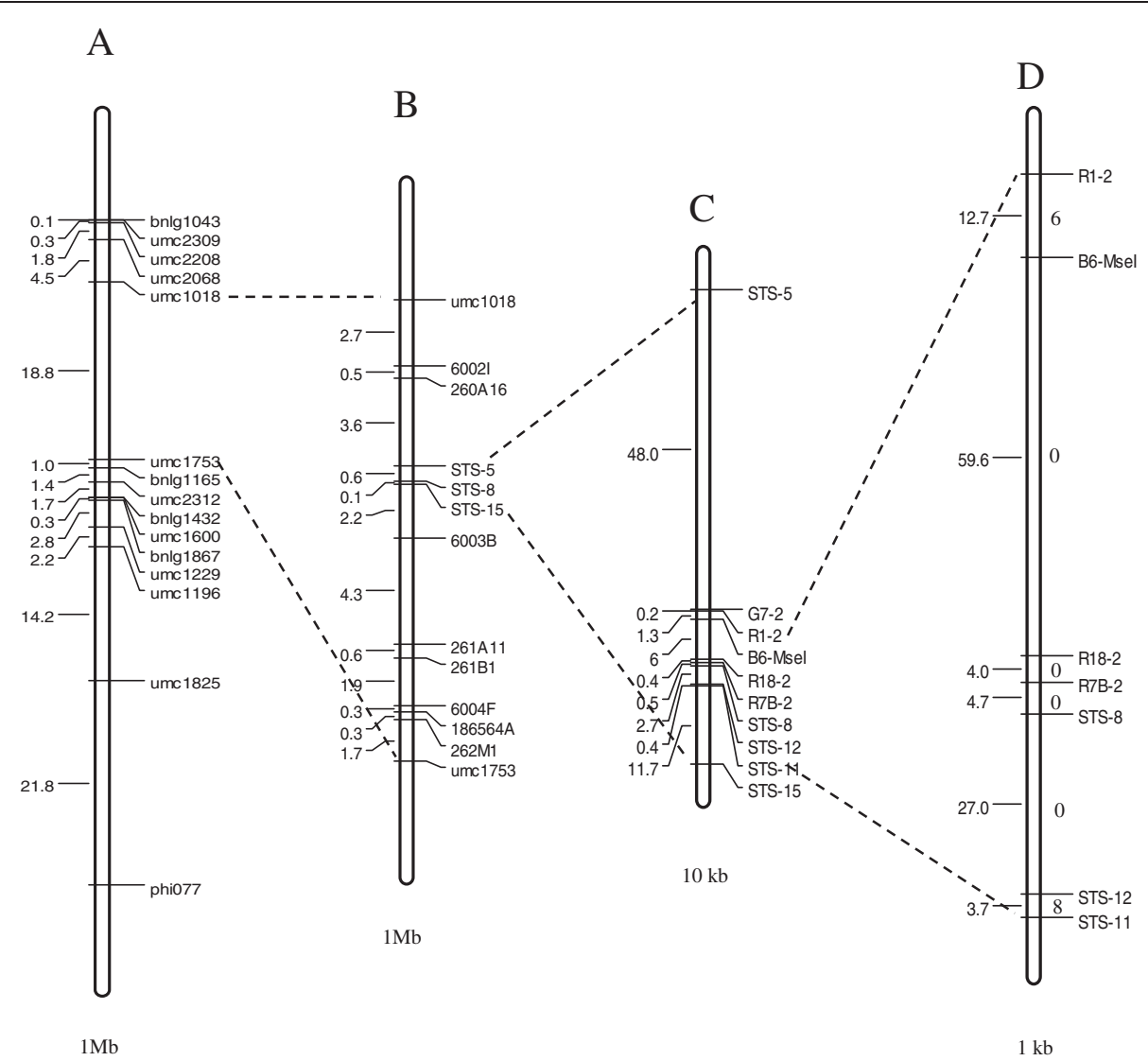

Figure 1 Diagram of the sequential fine-mapping process. (A) The physical map of 17 polymorphic SSR markers available from MaizeGDB (http://www.maizegdb.org/) in the Scmv1 genome region, 2 of the 17 markers with ambiguous location were deleted. By assaying 177 unselected $F_{2}$ plants, Scmv1 was narrowed to an $18.8 \mathrm{Mb}$ interval flanked by SSRs umc1018 and umc1573. (B) The physical map of the Scmv1 region after fine-mapping with 28 recombinants and 13 new developed markers. (C), (D) The final physical maps of Scmv1, estimated by genotyping 15 recombinants between STS-15 and STS-5 using eight newly developed markers. The number on the left side of the chromosome segments correspond to the physical distance of AGPv2, the units of $A, B, C$ and D were $1 \mathrm{Mb}, 1 \mathrm{Mb}, 10 \mathrm{~kb}, 1 \mathrm{~kb}$, respectively. The numerals on the right side of the chromosome segments represent the number of recombinants between two flanking markers.

marker, together with the R1-2 marker, were then used to jointly genotype all RILs at the Scmv1 locus. For each RIL population, four sets of 30 RILs, which differed at either the $S c m v 1$ or $S c m v 2$ or both loci, were separately selected for phenotypic evaluation.

In the RIL population of Zheng58 $\times$ Chang7-2, 28 of 30 RILs that share the same haplotype as Chang7-2 at both Scmv1 and Scmv2 loci (RR/RR) were highly resistant to SCMV. Of 30 RILs with the Chang7-2 haplotype at Scmv1, but the Zheng58 haplotype at Scmv2 (SS/RR), only one RIL was resistant to SCMV. Another two sets of 30 RILs sharing the same haplotype as Zheng58 at the Scmv1 locus (SS/SS and RR/SS) were all susceptible to SCMV. A similar result was observed in the RIL population of X178 $\times$ HuangC, all 30 RILs sharing the same haplotype as X178 at both Scmv1 and Scmv2 loci (RR/RR) displayed high resistance to SCMV. Of 30 RILs with the X178 haplotype at Scmv1 and HuangC haplotype at Scmv2 (SS/RR), only four RILs were resistant to SCMV. Another two sets of 30 RILs which share the same haplotype as HuangC at the Scmv1 locus (SS/SS or RR/SS) were all susceptible to SCMV. These findings indicate that the presence of both Scmv1 and Scmv2 loci are prerequisite for SCMV resistance as described in previous reports [13]. The significant difference $(P<0.01)$ in resistance percentages between RILs differing in haplotypes at both Scmv1 and Scmv2 loci suggested the presence of the same resistance loci in both RIL populations (Table 2).

\section{Further fine-mapping of Scmv1 in RILs}

Given the validation result, the flanking markers B4 and R1-2 were used to screen for recombinants within the 215.47-kb Scmv1 interval. Eight and nine recombinants were detected in the RIL populations Zheng58 $\times$ Chang7-2 and $\mathrm{X} 178 \times$ HuangC, respectively. For the Scmv2 locus, a co-segregating marker DFJ004 was used to determine the genotype for each RIL. All recombinants were repeatedly investigated for their resistance to SCMV under both greenhouse and field conditions. On the other hand, 
Table 1 New markers developed for fine mapping of the Scmv1 locus

\begin{tabular}{|c|c|c|c|c|c|}
\hline Name & BAC & Forward primer & Reverse primer & Type & Restriction enzymes \\
\hline 60021 & C0288C01 & GTTAGAACGTCTGCGCCTGT & TCCGTGCTGCGTAGTTACCT & STS & \\
\hline STS-5 & c0149g11 & GCAGGAGAAGAATCGGAGG & ACAAGAACAAGACAGCAGCG & STS & \\
\hline G7-2 & b0129G15 & AGTGTTCGTACCATGAGGTC & AATCCAGCCTCAGTAGAGTC & STS & \\
\hline $\mathrm{R} 1-2$ & b0129G15 & GCGTAGCAATATCCGTGTCA & TTCTGGTGTGAGCTTGAGCC & STS & \\
\hline R18-2 & b0598N23 & GATCTCGCAATCGTTGTAGC & CTCTCAATGCGGTAACCATC & STS & \\
\hline STS-8 & b0129G15 & ACCGATTATGTTCCATTGGC & GGTCCGACGCTCACTTCC & STS & \\
\hline STS-11 & b0598N23 & CAATAGACGTCGGACGAATG & ATGACATGCAACTACAGGCG & STS & \\
\hline STS-12 & b0598N23 & CTAACAAGCATGACGATCCG & AATTGCATCGATAAGCCACC & STS & \\
\hline STS-15 & b0606n15 & CTATTGGGCTTCCTTGTTCG & TATTCCTGTAGGCGACCTGG & STS & \\
\hline 261B1 & c0042B13 & AGCTTCCAAGTGTCCTTAGC & GCATCCTGGCTCAGCAATAC & STS & \\
\hline $6003 B$ & b0191N03 & CTGATGACTGCCACCATAGC & AACACGCTCGTGAGCAATAC & STS & \\
\hline $186564 \mathrm{~A}$ & b009012 & GTTCACGTTTCCCATGCTG & GCCACGTCGAACAACCTTAT & SSR & \\
\hline $261 \mathrm{~A} 11$ & c0361N19 & GAGAAGGCGAGAACGCATTG & AAGAGGCTACCGTAGGCGTC & SSR & \\
\hline $6004 \mathrm{~F}$ & b0390N08 & CGAGCTACTCAGCGTTGTCC & AAGAGGCTACCGTAGGCGTC & SSR & \\
\hline R7B-2 & b0598N23 & CACCGGAATAGTAGACGCAC & AGCTCTACTCCACCGGAACA & SSR & \\
\hline $260 A 16$ & C0115G19 & ATGTCTGCTGCCGTGAGTTC & CCTCCTGTCTGGTTGTCGTC & CAPS & Haelll \\
\hline B6-Msel & b0129G15 & GGATGAGAGGACTCTTGCAC & CTGCAAGCATGTCACAACA & CAPS & Msel \\
\hline $262 \mathrm{M} 1$ & C0043A01 & CAACTTCCATGACAGTGTCC & GTGTGATTCGGTGGCATAAC & CAPS & Mfel \\
\hline $\mathrm{RL}^{*}$ & 207B1-77 & GGATACAGCACCAAGGTTGA & AAGAGCTGTACACAGACGCC & SNP & \\
\hline SNP1* & 207B1-77 & GTAAGTGATAGGCGGAGTGG & AGGCTATCGTCGTGGATTGA & SNP & \\
\hline SNP2* & 207B1-77 & CCACTCTTGCTTCATCCTCA & AGTGTGTACGTGACCTTGATCT & SNP & \\
\hline SNP3* & 207B1-77 & AGATGGTGGTGAAGTGAAGG & CCTAGCAGGCTAGCACTGAT & SNP & \\
\hline SNP4* & 207B1-77 & GATGGAGTGCCGATTGCTAG & GGTCAACGAAGCCGATATTG & SNP & \\
\hline $\mathrm{R}^{*}$ & 207B1-77 & CACACACCACTTGCGATGTT & TCATGCGATGCCAGTGATAG & SNP & \\
\hline $2562 F^{*}$ & 207B1-77 & GCCGTATACAGGTCGAGCTT & GGAAGGAAGCCTAACTCGGA & SNP & \\
\hline $\operatorname{Trx}-1^{*}$ & 207B1-77 & CGATGCCGCCTAATATTCTC & GTAGCGGATCACGGATAACG & STS & \\
\hline $579 P 4^{*}$ & 207B1-77 & AACGCGAGTGCAGTAAGTCT & TGTTCCACAATGCTTTATCC & CAPS & Accll \\
\hline TC-4* & $579 B 1-75$ & CAGAGGAACAACAACCACCA & GCACATTACGGTTGAGTTGG & SNP & \\
\hline$B-4^{*}$ & $579 B 1-75$ & TACAAGTCAGGAGGTCCGGT & CTGGATGGTGTTGTGTCGAG & SSR & \\
\hline
\end{tabular}

*: Markers used for validation and further mapping in RILs, and these markers were developed based on the BAC sequences from 1145 inbred line.

through multiple comparisons of three resistant inbred lines, namely 1145, FAP1360A, and Huangzao4, and one susceptible B73 sequences within the B4/R1-2 interval, ten markers were developed to resolve the 17 recombinants.

Of the eight recombinants screened from the RIL population of Zheng58 $\times$ Chang7-2, four RILs were as resistant to SCMV as the resistant parent Chang7-2, whereas another four RILs were as susceptible to SCMV as the susceptible parent Zheng58. The Scmv2 locus in three of the susceptible recombinants, Nos.70, 683 and 1045, was identical to that in the susceptible parent Zheng58, and thus they could not be used to define the Scmv1 locus. The four resistant RILs, Nos. 413, 966, 246, and 841, together with one susceptible RIL No. 1012 had the resistance allele as Chang7-2 at the Scmv2 locus and thus could be used to fine-map the Scmv1 locus. The four resistant RILs had the Chang7-2 donor region upstream of the marker B4, and one susceptible RIL No. 1012 had the Chang7-2 donor region downstream of the marker SNP4. These data support that the Scmv1 locus is located upstream of the marker B4 (Figure 2A). The mapped Scmv1 region in the Zheng58 $\times$ Chang7-2 RIL population spanned the $112.39-\mathrm{kb} S \mathrm{cmv} 1$ region revealed by the near-isogenic $F_{2}$ population and was thus of no value to further narrow down the Scmv1 locus.

Among the nine recombinants screened from the RIL population $\mathrm{X} 178 \times$ HuangC, two displayed the same high resistance to SCMV as seen in the resistant parent X178, whereas another seven were as susceptible to SCMV as the parent HuangC. Three highly susceptible RILs, Nos. 1779, 1553, and 1746, carry the HuangC susceptibility allele at 
Table 2 Confirmation of the Scmv1 and Scmv2 regions in two RIL populations

\begin{tabular}{|c|c|c|c|c|c|c|}
\hline & & \multicolumn{4}{|c|}{ Genotype } & \multirow[t]{2}{*}{$P$-value } \\
\hline & & SS/SS & SS/RR & RR/SS & $\mathrm{RR} / \mathrm{RR}$ & \\
\hline \multicolumn{7}{|l|}{ Zheng58×Chang7-2 } \\
\hline & Resistant RILs & 0 & 1 & 0 & 28 & \multirow{2}{*}{$<0.01$} \\
\hline & Susceptible RILs & 30 & 29 & 30 & 2 & \\
\hline \multicolumn{7}{|l|}{ Huang CXX178 } \\
\hline & Resistant RILs & 0 & 4 & 0 & 30 & \multirow{2}{*}{$<0.01$} \\
\hline & Susceptible RILs & 30 & 26 & 30 & 0 & \\
\hline
\end{tabular}

The haplotype at the Scmv1 locus was detected by its flanked SSR markers R1-2 and B4; while the haplotype at the Scmv2 locus was identified by its co-segregating marker DJF004. The genotype SS/SS means both of the Scmv1 and Scmv2 loci are the same as Zheng58 or HuangC; RR/RR means both loci are the same as Chang7-2 or X178; RR/SS means Scmv2 and Scmv1are the same as Chang7-2/X178 and Zheng58/HuangC, respectively; while SS/RR means Scmv2 and Scmv1 are the same as Zheng58/HuangC and Chang7-2/X178, respectively.

$P$-value and $X^{2}$ were calculated by SAS 9.1 using two-way ANOVA.

the Scmv2 locus. They were thus excluded from finemapping of the Scmv1 locus. Four RILs, Nos. 1900, 1876, 2041, and 2062, had the X178 resistance allele at the Scmv2 locus. However, they were all susceptible to SCMV. This finding indicates the absence of the X178 resistance allele at the Scmv1 locus for these four RILs. Among them, Nos. 1876 and 1900 RILs had the donor HuangC region downstream of the marker R1-2, and Nos. 2041 and 2062 RILs had the donor HuangC region upstream of the marker SNP3, suggesting the Scmv1 locus is confined by the markers R1-2 and SNP3. Two highly resistant RILs, Nos. 2009 and 1523, carried the X178 resistance allele at the Scmv2 locus and their Scmv1 regions were flanked by the markers 579P4 and B4. Thus, the Scmv1 locus can be narrowed

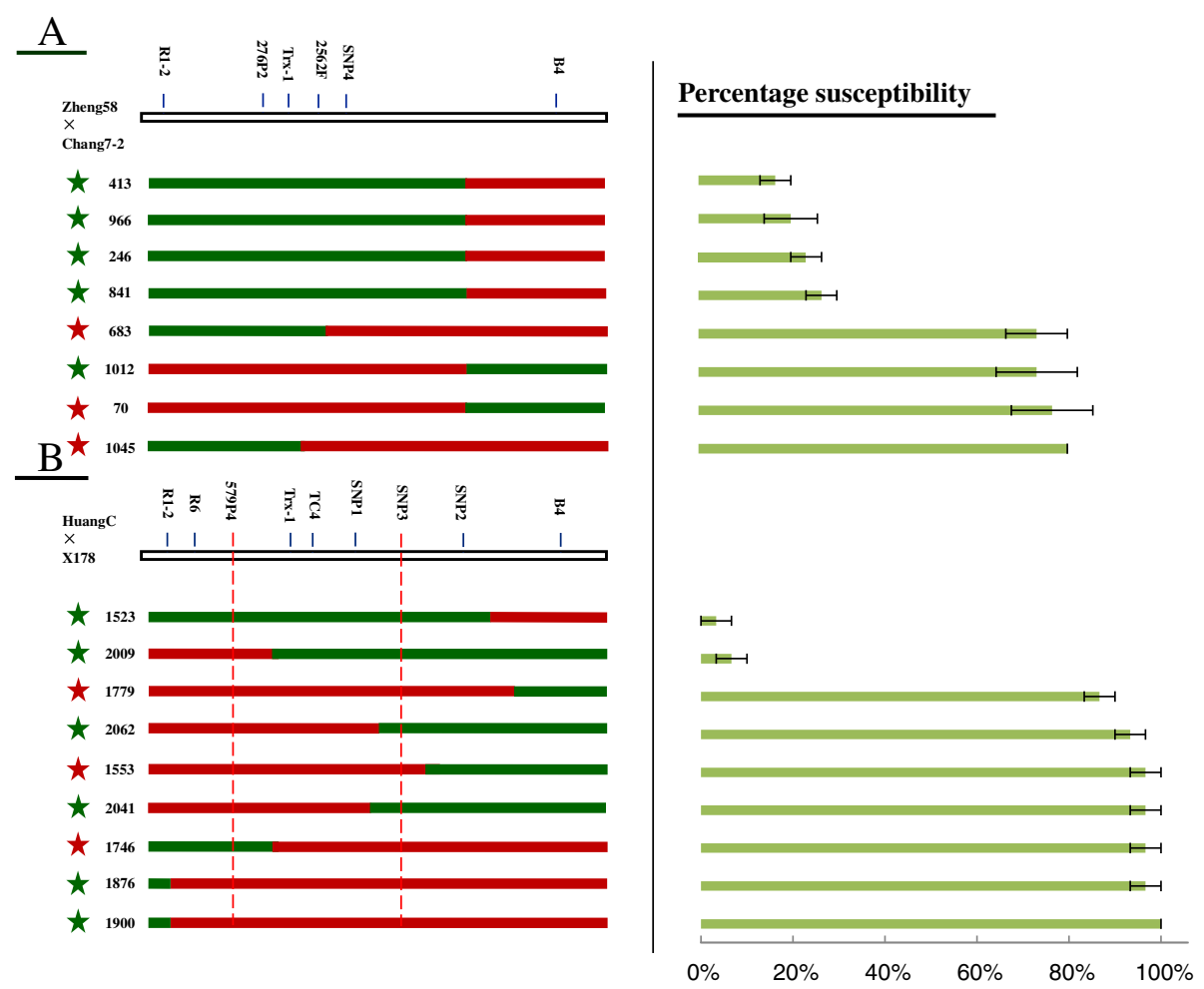

Figure 2 Further fine-mapping of Scmv1 in two RIL populations. The genetic structure for each recombinant type is depicted as green and red rectangles, (green symbolizes the fragment from resistant parents, chang7-2 or X178; red symbolizes the fragment from susceptible parents, Zheng58 or HuangC). The star represents allele at the Scmv2 locus on chromosome 3 (green symbolizes resistance allele from Chang7-2 or X178; red symbolizes susceptibility allele from Zheng58 or HuangC). The light-green bars on the right represent the average percentage of plants with SCMV symptoms based on three independent experiments in greenhouse. A) The Scmv1 locus can be delimited to a 215.47-kb region in RIL population Zheng58xChang7-2. B) The Scmv1 locus can be delimited to a 59.21-kb region flanked by markers $579 P 4$ and SNP3 (in red dash-dot lines) in RIL population HuangCXX178. 
down into a 59.21-kb region flanked by the markers 579P4 and SNP3 (Figure 2B), located within the 112.39$\mathrm{kb}$ interval defined by the near-isogenic $\mathrm{F}_{2}$ population.

\section{Predicting putative genes within and around the mapped Scmv1 region}

A 215.47-kb sequence flanked by the markers B4 and R1-2 was retrieved from www.maizesequence.org to identify candidate $S c m v 1$ genes. There are still seven gaps left in this region, indicating some missing sequences. Transposable elements account for $55.27 \%$ of this region, of which $82.28 \%$ are retrotransposons, and $99.87 \%$ of the retrotransposons are long terminal repeat elements (data not show). We used Fgenesh software (http://linux1. softberry.com/berry.phtml, version 2.6) to predict ten putative genes from the masked sequence, including seven genes with putative functions, two hypothetical genes, and one gene without significant similarity to known genes (Figure 3C). No typical resistance $(R)$ gene was found in the $S c m v 1$ region. Analysis of possible functions for the seven predicted genes with Gene Ontology (GO) and Kyoto Encyclopedia of Genes and Genomes (KEGG) annotation indicated three of the genes were likely to confer resistance to SCMV (Table 3).

Two of the predicted genes are cycloartenol synthase1like (CAS1-like) genes with high sequence similarity to genes from Arabidopsis thaliana. The first had the coverage of $76 \%$ and E-value of $1.00 \mathrm{E}-129$, whereas the second had the coverage of $60 \%$ and E-value of $1.00 \mathrm{E}-74$. These two CAS1-like genes have $74.18 \%$ similarity $(204 / 275)$, with an E value of 2.00E-117, although they differ in the lengths of their amino-acid sequences. We designated the larger gene (403 residues) as CAS1-like-1, and the smaller one (360 residues) as CAS1-like-2. The other candidate gene, Zmtrx-h, encodes a putative 119 -residue protein that is highly similar to thioredoxin (100\% coverage; $\mathrm{E}=4.00 \mathrm{E}-64)$, an oxidoreductase that acts on sulfur groups of donors, using disulfide as an acceptor.

Integration of the mapping results indicated that Scmv1 localizes to a $59.21-\mathrm{kb}$ region flanked by $579 \mathrm{P} 4$ and SNP3 (Figure $3 \mathrm{~B}$ ). Of the five predicted genes located in this region, $C A S 1$-like- 2 and $Z m t r x-h$ are more likely than the other three genes to confer resistance to SCMV.

\section{Candidate gene-based association mapping of the Scmv1 locus}

To verify the candidate region and possibly refine the analysis by identifying quantitative trait nucleotide polymorphisms, we conducted an association mapping study using a panel of 94 inbred lines (Additional file 2), which were divided into six subgroups based on 70 SSR markers (Additional file 3). Multiple sequence alignment analysis identified 30 polymorphisms in six informative amplicons. Linkage disequilibrium (LD) analysis revealed lower $\mathrm{r}^{2}$ between these polymorphisms (Figure 4), with $91.03 \%$ of the $r^{2}<0.20$. Two (single nucleotide polymorphism) SNP sites, S454 and S126, separately located on the 3 '-end and intron of Zmtrx- $h$ were significantly associated with SCMV resistance $(P<0.05$; Table 4 and Additional file 4). Nonetheless, a low $\mathrm{r}^{2}$ value of 0.01 indicated two independent functional variants.

Given that 19 of the 94 maize inbred lines, including Mo17, could not be amplified by polymerase chain reaction (PCR) using any of the 17 primer pairs scattered throughout the Scmv1 region, we were able to identify a large PAV covering the Scmv1 region. This PAV was verified by Springer et al. [23], who reported a $2.6-\mathrm{Mb}$ region on chromosome 6 including the $S c m v 1$ region present in the B73 genome but not in Mo17. Strikingly, all of the 19 inbred lines were susceptible to SCMV, resulting in a strong association with SCMV resistance $(P=9.96 \mathrm{E}-4)$.

\section{Discussion}

Precise phenotypic evaluation is crucial for marker-trait association analysis [24-27], especially for those traits with incomplete penetrance and phenotypic plasticity. Apart from being influenced by two major genes, resistance of maize to SCMV is also affected by genetic background and various environmental factors $[13,17,18]$. To obtain reliable phenotypic data, we developed a $\mathrm{F}_{2}$ mapping population from a near-isogenic pair where the resistance allele was fixed at the Scmv2 locus, and only Scmv1 was segregating [28]. Furthermore, selection of only susceptible individuals from the $\mathrm{F}_{2}$ population for fine-mapping of the $S c m v 1$ locus reduced the possibility of misclassification, as heterozygotes tend to be more plastic in their response to SCMV inoculation.

Advances in multiple genomic platforms and analytical methods allow fine-mapping and cloning of QTL responsible for important agronomic traits [29]. In the current study, development of polymorphic markers in the 112.39-kb Scmv1 region was a challenge due to highly repetitive sequences. We thus sequenced the positive BAC clones from the 1145, FAP1360A, and Huangzao4 BAC libraries to mask the repetitive sequences at the Scmv1 locus, in order to reveal single/low-copy sequences for marker development. Generally, re-sequencing of more maize inbred lines [30] and the availability of additional publicly accessible sequence information (such as for Mo17: http://www.phytozome.net/maize. php; and Palomero: http://www.palomerotoluqueno. org/index.php) will facilitate the development of markers in targeted genome regions. During the process of finemapping, we found that the published order of BAC contigs (http://www.maizesequence.org) for the $S c m v 1$ region is incorrect. The BAC-based B73 whole-genome physical map suggests that the adjacent BAC contigs \#260 and \#261 should be physically close to one another. However, the 


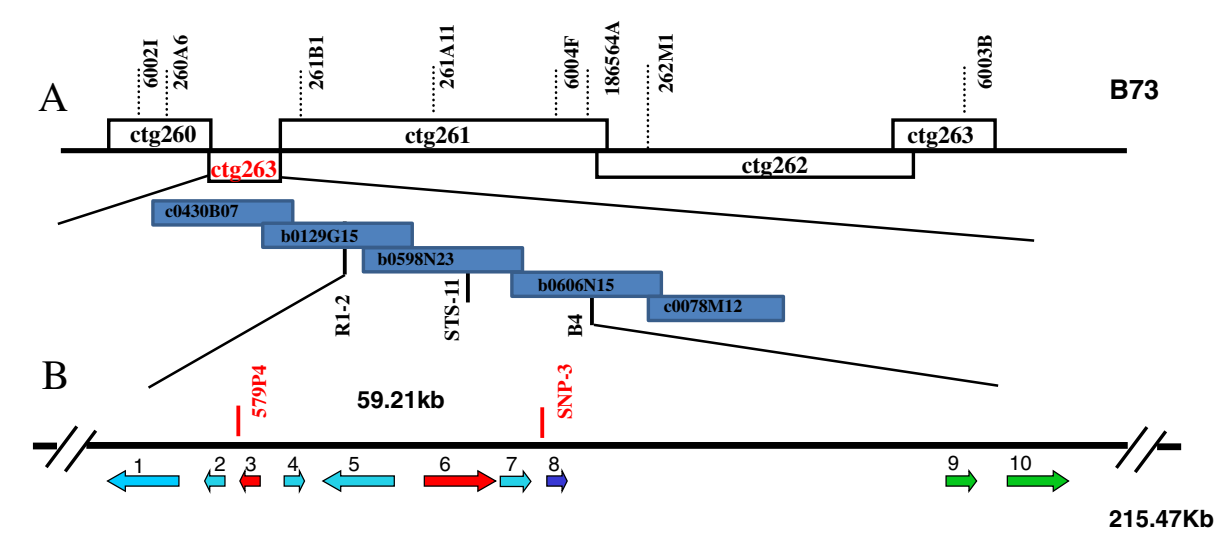

$\begin{array}{ll}\Rightarrow \text { Candidate genes } & \Rightarrow \text { Genes with putative function } \\ \Rightarrow \text { Hypothetical protein } & \Rightarrow \quad \text { No significant similarity }\end{array}$

Figure 3 Integration of the mapping results from three populations. (A) The combined mapping of Scmv1. The revised order of contigs on the Scmv1 region is \#260-\#263-\#261-\#262-\#264 (ctg263 in red is where Scmv1 located). Scmv1 was finally assigned to a 59.21-kb region flanked by markers 579P4 and SNP3. (B) Gene prediction of a 215.47-kb B73 sequence covering the targeted region: each arrow stands for a predicted gene, the head represents gene orientation and the length of each arrow corresponds to gene length (detailed information listed in Table 3).

genetic distance is so large that none of the recombinants within the umc1018/umc1753 interval could be further resolved by the newly developed markers based on the contig \#261. Only the markers developed from the contig \#263 could resolve the recombinants. This indicates that contig \#263 is located between contigs \#260 and \#261, and that the correct contig order must be \#260-\#263-\#261-\#262\#264 (Figure 3A). This correct contig order has been released (www.maizesequence.org).

Searching for recombinants with an unambiguous phenotype is crucial for fine-mapping. Thus, we used only highly susceptible individuals in the $\mathrm{F}_{2}$ mapping population for fine-mapping, which allowed us to fine-map the Scmv1 locus into a 112.39-kb region flanked by R1-2 and STS-11. From the fact that the Scmv1 locus has been repeatedly detected $[8,12,14,21]$, we inferred that $S c m v 1$ is likely present in various resistant inbred lines. Based on this hypothesis, two RIL populations were used to fine-map the $S c m v 1$ locus into a 59.21-kb region flanked by the markers $579 \mathrm{P} 4$ and SNP3.

The public B73 sequence suggested that two predicted genes, the CAS1-like homolog and Zmtrx-h gene, are the most likely candidates for Scmv1 owing to their potential roles in disease defense response [31,32], although the potential influence of hypothetical proteins in the Scmv1 region on SCMV resistance cannot be excluded. Comparison with the complete CAS1 gene from Arabidopsis thaliana, which encodes 759 residues,

Table 3 Analysis of genes predicted from the repeat-masked sequence between the 215.47-kb interval of the markers B4/R1-2

\begin{tabular}{|c|c|c|c|c|c|}
\hline Predicted gene & Length of gene & First hit & E-value & Query coverage & KEGG entry \\
\hline 1 & 403aа & putative cycloartenol synthase & $1.00 \mathrm{E}-129$ & $76 \%$ & AT2G07050 \\
\hline 2 & 137aа & 605 ribosomal protein L24 & 7.00E-04 & $16 \%$ & AT3G53020 \\
\hline $3^{*}$ & 119aа & thioredoxin H-type & 4.00E-64 & $100 \%$ & AT5G39950 \\
\hline 4 & 239aа & putative transposable element & $2.00 \mathrm{E}-29$ & $89 \%$ & AT3G22220 \\
\hline $5^{*}$ & 360aа & putative cycloartenol synthase & $1.00 \mathrm{E}-74$ & $60 \%$ & AT2G07050 \\
\hline 6 & 618aа & sucrose synthase 2 & 4.00E-52 & $36 \%$ & AT4G02280 \\
\hline 7 & $342 \mathrm{aa}$ & salT gene product & $3.00 \mathrm{E}-20$ & $100 \%$ & AT1G19715 \\
\hline 8 & 93aa & no significant similarity & NA & NA & NA \\
\hline 9 & 330aа & hypothetical protein cauri_1913 & $3.30 \mathrm{E}+00$ & $53 \%$ & NA \\
\hline 10 & 455aа & hypothetical protein & $2.00 \mathrm{E}-107$ & $98 \%$ & NA \\
\hline
\end{tabular}

*stands for candidates. First hit, E-value, and Query coverage were BLASTP results of predicted genes from NCBI. KEGG entry is the search word for pathway information about the predicted genes based on GO blast search results. NA means not available. 


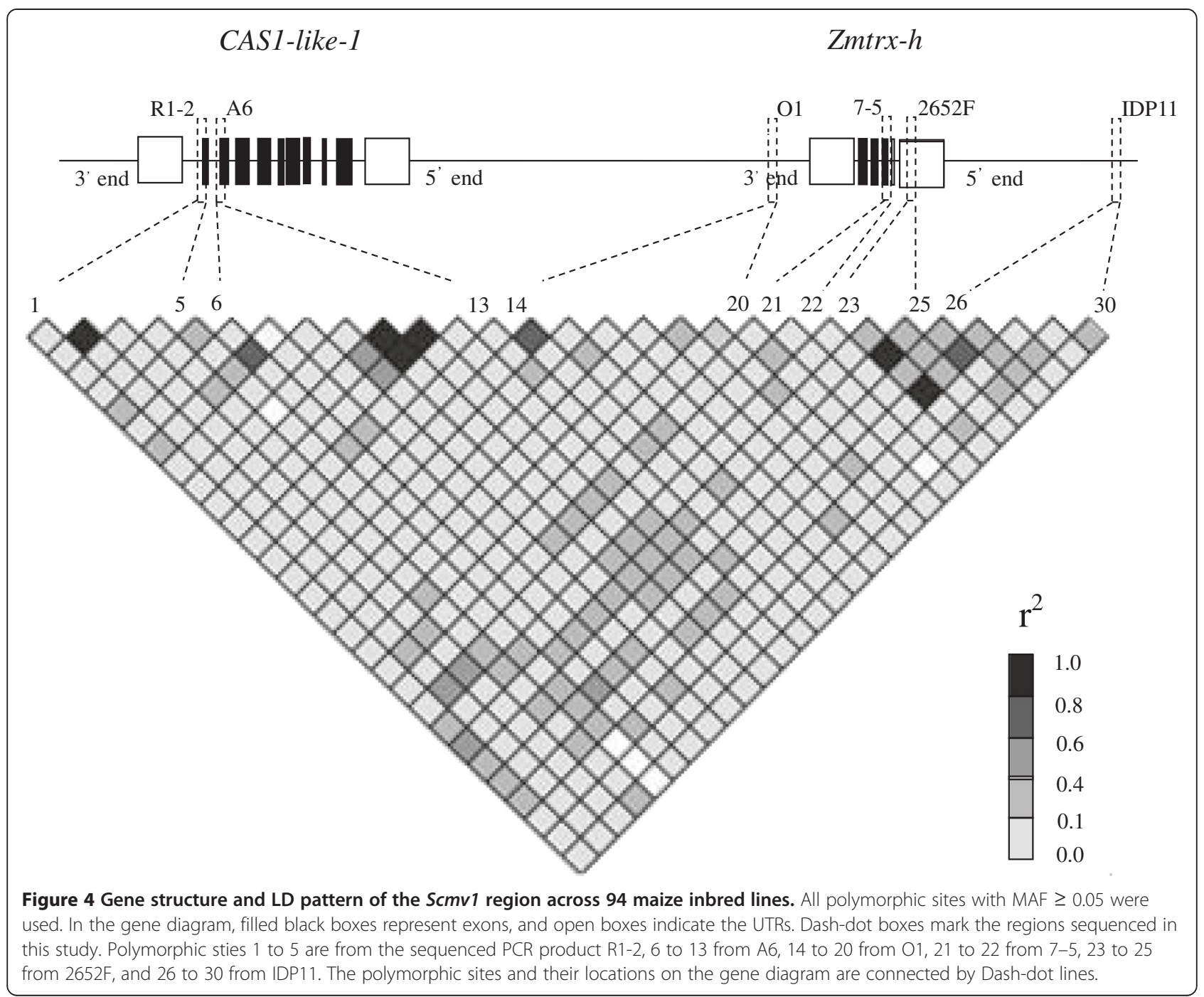

suggests that the putative CAS1-like gene may be partial gene containing the TERPENE_SYNTHASES motif. CAS1 is involved in biosynthesis of steroids and nonsteroidal secondary metabolites, such as campesterol and stigmasterol [31]. Campesterol is the precursor of brassinosteroids, an important class of plant hormones that functions in cellular signal transduction, whereas the accumulation of stigmasterol stimulates an important plant metabolic process that occurs following bacterial infection of leaves [32]. Given that phenotypic variance associated with changes in the $S c m v 1$ region are influenced by the stage of plant development [19], it remains to be established whether the situation with Scmv1 closely resembles that of $H m 2$. a truncated duplicate of $H m 1$. Compared to $H m 1, H m 2$ preferentially functions in adult plants, conferring resistance against the leaf spot and ear mold disease caused by Cochliobolus carbonumrace 1 [33,34].

Thioredoxin is a master regulator of cellular redox status [35], with h-type thioredoxin reported to function in defense responses to viruses [36] and fungi [37]. Given that the Zmtrx-h protein in the Scmv1 region lacks the conserved WC(G/P)PC motif, which is essential for redox protein activities, it seems unlikely that Zmtrx-h affects cellular redox status. Hence, additional investigation of the roles of the three candidate genes is required to uncover the mechanism of SCMV resistance.

Linkage and association analysis are two prevalent approaches to map genes or QTL. Both can be used in a complementary manner to dissect the genetic basis of traits of interest [38,39], as well as to fine-map causative variants of targeted QTL [40]. Six pairs of primers were used to test the association of specific stretches of sequence in the Scmv1 region with SCMV resistance. General linear model (GLM) analysis highlighted three polymorphisms associated with SCMV resistance. The significant association between the PAV and SCMV resistance validates the role of the $S c m v 1$ region with disease resistance, whereas the association between two 
Table 4 Associations between SCMV resistance and polymorphic sites in the Scmv1 genome region

\begin{tabular}{|c|c|c|c|c|c|c|}
\hline \multirow[t]{2}{*}{ Sites } & \multirow[t]{2}{*}{ Location } & \multirow[t]{2}{*}{ Alleles $^{a}$} & \multirow[t]{2}{*}{ Frequency } & \multicolumn{2}{|c|}{ P-value } & \multirow{2}{*}{$\begin{array}{c}\text { FAP1360A } \\
\text { F7 }^{d}\end{array}$} \\
\hline & & & & GLM $^{\mathbf{b}}$ & $S M A^{c}$ & \\
\hline S454 & 3'-end of Zmtrx-h & $T / G$ & $15 / 50$ & 0.0145 & 0.0039 & $\mathbf{G} / T$ \\
\hline S126 & Intron of Zmtrx-h & $\mathrm{T} / \mathrm{C}$ & $5 / 68$ & 0.0371 & 0.0281 & $\mathrm{C} / \mathrm{C}$ \\
\hline PAV & Bin 6.01 & $0 / 1$ & $19 / 75$ & $9.56 \mathrm{E}-4$ & $2.07 \mathrm{E}-5$ & $1 / 1$ \\
\hline
\end{tabular}

S454 was identified from the marker 01, while S126 was identified from the marker 7-5.

atetters (or number) in bold indicate the favourable alleles.

${ }^{b}$ P-value was calculated using GLM in TASSEL 2.0.

${ }^{\mathrm{C}} \mathrm{P}$-value was calculated using single marker analysis.

${ }^{\mathrm{d}}$ The alleles of inbred lines FAP1360 and F7.

other SNPs and SCMV resistance provides statistical support for $Z m t r x-h$ as the primary candidate for $S c m v 1$. Moreover, these two SNP located in the intron and 3 '-end of $Z m t r x-h$, respectively, seemingly having an influence on the gene's expression. The CAS1-like-2 gene cannot yet be excluded, as there was no primer pair available in the vicinity of it. Therefore, a complementary functional test via transformation is still needed to identify which candidate gene, if any, defines Scmv1. These transgenic experiments are underway in our lab.

Broad-spectrum resistance (BSR), resistance against two or more types of pathogen species or the majority of races of the same pathogen species [41], is essential to improve the resistance of crops to various diseases. The quantitative resistance gene $\operatorname{Lr} 34$ cloned recently confers resistance to leaf rust, stripe rust, powdery mildew and various other diseases of wheat [42]. The evidence for the presence of multiple disease resistance genes in a maize association population [43] revealed that quantitative BSR can be conferred by a single gene. The Scmv1 region has been reported to associate with resistance to other members of the Potyviridae family, including WSMV and MDMV $[44,45]$. The near isogenic line $F 7^{\mathrm{RR} / \mathrm{RR}}$, which was used as a resistant parental line in this study, was also found to be resistant to MDMV and ZeMV [28]. According to a model proposed by Kou [46], a single gene conferring BSR may function in either basal-resistance pathways, in overlapping pathways that confer race-specific resistances, or at sites of cross-talk between different defense pathways. Given that both candidate genes have putative roles in basal resistance, Scmv1 likely confers BSR, which would be advantageous in environments where plants are threatened by multiple pathogens. However, this speculation remains to be verified by artificial inoculation of transgenic plants and recombinants with the appropriate range of pathogens.

\section{Conclusion}

We have fine-mapped $S c m v 1$ to a 59.21 -kb region through integrating mapping data from different populations. This provides indispensable data for initiating molecular breeding to improve SCMV resistance in maize, a crop that is critical to world food security. We identified two candidates, of which the Zmtrx-h gene seems the most promising. Map-based cloning of Scmv1 and transgenic analyses will shed light on the molecular mechanism of this important plant-virus interaction.

\section{Methods}

\section{Populations used for fine-mapping}

The F7 near-isogenic lines, F7 ${ }^{\mathrm{RR} / \mathrm{RR}}, \mathrm{F} 7^{\mathrm{RR} / \mathrm{SS}}$, and $\mathrm{F} 7^{\mathrm{SS} / \mathrm{RR}}$, derived from two early-maturing European maize inbred lines, FAP1360A (completely resistant to SCMV) and F7 (highly susceptible to SCMV), have been developed as described by Ingvardsen [17] (Additional file 5). A total of $2,200 \mathrm{~F}_{2}$ plants derived from the cross of $\mathrm{F} 7^{\mathrm{RR} / \mathrm{RR}} \times \mathrm{F} 7^{\mathrm{RR} / \mathrm{SS}}$ were collected as a mapping population for fine-mapping of Scmv1. Initially, a panel of 177 plants, randomly selected from the $F_{2}$ mapping population, was used as an unselected mapping population to delimit the Scmv1 region. Each plant was mechanically inoculated with SCMV twice and scored at a weekly interval to evaluate resistance to SCMV. Additional markers in the Scmv1 region were developed to genotype the unselected $F_{2}$ population. In parallel, the remaining 2,023 $\mathrm{F}_{2}$ plants were investigated for their resistance to SCMV, resulting in the identification of 510 plants with typical SCMV symptoms indistinguishable from their susceptible parent F7. These 510 susceptible $\mathrm{F}_{2}$ plants, together with 28 recombinants from the unselected $F_{2}$ mapping population, formed the $F_{2}$ sub-population for fine-mapping of Scmv1.

\section{Validation of the Scmv1 locus in two RIL populations}

Two single-seed descent $F_{6}$ RIL populations were employed to validate and further fine map the $S c m v 1$ locus. One was developed from the cross between Zheng58 (susceptible to SCMV) and Chang7-2 (resistant to SCMV), a commercial hybrid (ZD958) that is currently widely cultivated in China. The other RIL population was derived from the cross between X178 (completely resistant to SCMV) and HuangC (highly susceptible to SCMV), another commercial hybrid (ND108) widely grown in China. A total of 2,206 $\mathrm{F}_{6}$ RILs (1,285 from Zheng58 $\times$ Chang7-2 and 921 from $\mathrm{X} 178 \times$ HangC) were planted in the experimental station at 
Shangzhuang, Beijing, during the summer of 2010. All RILs were genotyped using the markers R1-2 and B4 flanking the Scmv1 locus, as well as a co-segregating marker DJF004 at the Scmv2 locus [17]. For each RIL population, four sets of 30 RILs, which differed at the Scmv1 or Scmv2 locus or both loci, were separately selected for phenotypic evaluation, whereas all recombinants between R1-2 and B4 were investigated for their SCMV resistance.

\section{Artificial inoculation}

Seeds were sown in a soil/vermiculite mixture in $0.80-\mathrm{L}$ pots maintained under greenhouse conditions. Virus inocula (Chinese isolate) were prepared from the SCMV-infected susceptible F7 plants. Young leaf tissue with typical mosaic symptoms was homogenized in five volumes of $0.01 \mathrm{M}$ sodium phosphate buffer, $\mathrm{pH}$ 7.0. Carborundum was added to the sap to facilitate rubinoculation. All leaves of each plant were rub-inoculated at the three- to four-leaf stage. One week after inoculation, all the symptomless plants were re-inoculated to avoid any escape. During sap preparation and mechanical inoculation, the inocula were put on ice to maintain low-temperature conditions.

For phenotypic evaluation of inbred lines and selected RILs, 12 kernels of each line were planted in four $0.80-\mathrm{L}$ seeding pots. Whereas 10 plants were rub-inoculated with virus inoculum, two plants were not inoculated. These RILs were evaluated along with their parent lines as positive and negative controls for all tests. RILs with the same symptoms $(P>0.05$, t-test $)$ as the susceptible parent or the resistant parent were regarded as susceptible (S) or resistant (R). Three independent experiments were performed during September 2010 to July 2011 to ensure accurate phenotyping.

\section{DNA extraction and molecular analyses}

Immature leaf samples were harvested from each individual and immediately frozen in liquid nitrogen. DNA was extracted using the SDS method described by Murray and Thompson [47].

Apart from new markers developed from public BAC sequences, all SSR markers were retrieved from the Maize Genetics and Genomics Database (www.maizegdb.org) (Additional file 1) and synthesized by Invitrogen (Beijing, China). To design specific markers for fine-mapping, we first anchored the QTL in bin 6.01 to the B73 BAC-based whole-genome physical map (http://www.maizesequence. org/) using flanking SSR markers. We next extracted BACs, expressed sequence tags, and Indel polymorphic sequences (http://www.ncbi.nlm.nih.gov/; http://magi.plantgenomics. iastate.edu/browseMarkers.do) available in the targeted region to develop new polymorphic markers (SSR, STS, SNP, and CAPS). The retrieved sequences were analyzed with RepeatMasker (http://www.repeatmasker.org/cgi-bin/ WEBRepeatMasker) followed by BLAST analysis against maize high-throughput genome sequence and genome sequence survey to remove redundant sequences [48]. All primers for the single/low-copy sequences were designed using the software PRIMER 5.0 with the criteria that the primer length should be 20 nucleotides, with a $40-60 \%$ GC content, no secondary structure, and no consecutive tracts of the same nucleotide. The microsatellite search tool SSRHunter1.3 [49] was used to mine SSR sequences of 4-6 tandem copies of $>2$ base pairs.

For PCR-mediated detection of SSR and STS markers, reactions were prepared in a $10-\mu \mathrm{L}$ reaction volume containing $100 \mathrm{ng}$ of genomic DNA, $1 \mu \mathrm{L}$ of $10 \times$ PCR buffer, $0.2 \mathrm{mM}$ of each dNTP, $0.2 \mu \mathrm{M}$ of each forward and reverse primer, and $1 \mathrm{U}$ of Taq polymerase. To detect SNP and CAPS markers, $50 \mu \mathrm{L}$ reactions contained $300 \mathrm{ng}$ of template DNA, $5 \mu \mathrm{L}$ of $10 \times$ PCR buffer, $1 \mathrm{mM}$ of each $\mathrm{dNTP}, 1 \mu \mathrm{M}$ of each forward and reverse primer, and $5 \mathrm{U}$ Taq polymerase (Beijing Transgen Biotech Co.LTD). All PCR reactions were performed using a PTC-200 Peltier Thermal Cycler (MJ Research Inc., Canada) with particular annealing temperature and elongation duration adapted to each reaction. The PCR products were subjected to electrophoresis using either 1\% agarose gels (visualized using a gel imaging system from BioRad Laboratories Inc., USA) or $6 \%$ polyacrylamide gels (amplification products were visualized by silver staining). SNP markers were sequenced at the Beijing Genomic Institute.

\section{Analysis of candidate genes}

To predict candidate genes, we retrieved the DNA sequence of the Scmv1 region from the B73 wholegenome sequence (http://www.maizesequence.org) for candidate gene prediction. The repetitive sequence in the Scmv1 region was analyzed by RepeatMasker version 3.3.0 (http://www.repeatmasker.org). We used the Fgenesh program (http://linux1.softberry.com/berry.phtml, version 2.6) to predict genes present in the single/lowcopy Scmv1 region. For functional analysis, the deduced amino-acid sequence of each putative gene was compared against the "nr" database via BLASTP on National Center of Biotechnology Information website (http://blast.ncbi.nlm.nih.gov/). Detailed GO and KEGG annotation was applied to the gene sequences predicted by BLAST searches (http://www.geneontology.org/ and http://www.genome.jp/kegg/).

\section{Association mapping of the Scmv1 region}

A total of 94 maize inbred lines were phenotyped in two distinct experiments (October 2007, May 2010) under greenhouse conditions using the same artificial inoculation 
procedure as RIL populations. The same 94 inbred lines were genotyped using a set of 70 SSR markers that were evenly distributed throughout the maize genome (Additional file 6). Based on the B73 sequence and using Primer 3 (http://frodo.wi.mit.edu/) or Premier 5.00 (PREMIER Biosoft International, USA), we designed 17 pairs of PCR primers (Additional file 7) flanking the three candidate genes. We used these primers to first screen for polymorphisms between F7, FAP1360A, Zheng58, Chang7-2, HuangC, and X178. Most primer pairs were discarded because of unreliable amplification. Six primer pairs with polymorphic products were used to amplify these 94 inbred lines to identify potential polymorphic sites. Of these six polymorphic amplicons, two and three are located in the vicinity of CAS1-like-1 and Zmtrx-h, respectively, whereas no primer pair around CAS1-like-2 was available. Nucleotide sequences alignments were done using the multiple sequence alignment software MUSCLE [50] and subsequently refined manually. Q was inferred using STRUCTURE 2.2 [51,52] with 70 SSR markers. Five independent runs were performed setting the number of populations (k) from 1 to 10, burn in time and MCMC (Markov Chain Monte Carlo) replication number both to 500,000, and a model for admixture and correlated allele frequencies. The $k$ value was determined by $\operatorname{LnP}(\mathrm{D})$ in STRUCTURE output and an ad hoc statistic $\Delta \mathrm{k}$ based on the rate of change in $\operatorname{LnP}(\mathrm{D})$ between successive $k$. Lines with membership probabilities $\geq 0.75$ were assigned to corresponding clusters; lines with membership probabilities $<0.75$ were assigned to a mixed group. Groups were further subdivided into subgroups using a similar methodology. The runs most consistent with breeder's knowledge about pedigree were used to assign lines into clusters. The levels of LD between two sites were calculated using TASSEL2.0 [53]. The associations between the extracted polymorphic sites with Minor Allele Frequency (MAF) $\geq 0.05$ and SCMV resistance were carried out using GLM in TASSEL2.0.

\section{Additional files}

\section{Additional files 1: The list of public SSR markers for mapping of Scmv1.}

Additional files 2: The 94 maize inbred lines and their resistance performance to SCMVR means resistance to SCMV, while $S$ means susceptible to SCMV.

Additional files 3: Population structure of 94 maize inbred lines estimated using 70 SSRs. Population structure was assessed by STRUCTURE. Each individual is represented by a vertical bar, partitioned into colored segments with the length of each segment representing the proportion of the individual's genome from $\mathrm{k}=6$ groups. For all classes, a given group is represented: red, REID; Blue, P; green, LAN; Yellow, TSPT; pink, Z330; turquoise, OTHER.

Additional files 4: Associations between SCMV resistance and polymorphic sites in the Scmv1 genome region. a: P-value calculated using GLM. \#: polymorphic sites associated with SCMV resistance.
Additional files 5: The procedure of developing the Scmv1 mapping population.

Additional files 6: List of SSR markers for structure and kinship analysis.

Additional files 7: Primers designed for association mapping. *: The PCR products amplified by these primers contained polymorphic sites that could be used for association mapping.

\section{Abbreviations}

BAC: Bacterial artificial chromosome; BSR: Broad-spectrum resistance; CAS: Cycloartenol synthase1-like; GO: Gene ontology; K: Kinship; KEGG: Kyoto encyclopedia of genes and genomes; LD: Linkage disequilibrium; MAF: Minor Allele Frequency; JGMV: Johnson grass mosaic virus; GLM: General linear model; PCR: Polymerase chain reaction; Q: Population structure; PAV: Presence/absence variations; QTL: Quantitative trait loci;

RIL: Recombinant inbred line; SCMV: Sugarcane mosaic virus; SNP: Single nucleotide polymorphism; SrMV: Sorghum mosaic virus; SSR: Simple sequence repeat; WSMV: Wheat streak mosaic virus; ZeMV: Zea mosaic virus.

\section{Competing interests}

The authors declare that they have no competing interests.

\section{Authors' contributions}

$\mathrm{L}, \mathrm{QL}, \mathrm{CRI}$ and UKF performed mapping experiment in segregating population. BW and JL provided two RIL populations. YT, QL, carried out fine-mapping in these two RIL populations. YZ, RZ and YT completed the association mapping work. MX and TL supervised the research, designed the experiments and were involved in data analysis. YT wrote the manuscript draft, MX and TL edited and revised the manuscript. All authors read and approved the final manuscript.

\section{Acknowledgements}

This study was financially supported by the NSFC (31371625), Ministry of Agriculture of China (2011ZX08009-003-001), National High-tech and Development Program of China (2012AA10A306 and 2012AA101104). We thank Dr. Xiaohong Yang at the China Agricultural University for critical guidance of association analysis in this study.

\section{Author details}

'National Maize Improvement Center, China Agricultural University, 2 West Yuanmingyuan Road, Beijing 100193, People's Republic of China.

${ }^{2}$ Department of Agronomy, lowa State University, 1204 Agronomy Hall, Ames, lowa 50011, USA. ${ }^{3}$ Research Center Flakkebjerg, University of Aarhus, 4200, Slagelse, Denmark.

Received: 1 August 2013 Accepted: 9 October 2013

Published: 18 October 2013

\section{References}

1. Fuchs $\mathrm{E}$, Gruntzig M: Influence of sugarcane mosaic-virus(SCMV) and maize-dwarf mosaic-virus(MDMV) on the growth and yield of 2 maize varieties. Zeitschrift Fur Pflanzenkrankheiten Und Pflanzenschutz-Journal of Plant Diseases and Protection 1995, 102(1):44-50.

2. Wang ZH, Li XH, Yuan LX, Han XQ, Zhang SH: Genetic diversity of elite maize germplasm for resistantance to SCMV. Acta Agron Sin 2003, 29(3):391-396.

3. Trzmiel K: First report of sugarcane mosaic virus infecting maize in Poland. Plant Dis 2009, 93(10):1078-1078.

4. Marie-Jeanne V, Hariri D, Doucet R, Signoret PA: First report of sugarcane mosaic virus on maize in the centre region of France. Plant Dis 2011, 95(1):70-70.

5. Shukla DD, Ward CW: Identification and classification of potyviruses on the basis of coat protein-sequence data and serology. Arch Virol 1989, 106(3-4):171-200.

6. Kuntze L, Fuchs E, Gruntzig M, Schulz B, Klein D, Melchinger AE: Resistance of early-maturing European maize germplasm to sugarcane mosaic virus (SCMV) and maize dwarf mosaic virus (MDMV). Plant Breed 1997, 116(5):499-501. 
7. Seifers DL, Salomon R, Marie-Jeanne V, Alliot B, Signoret P, Haber S, Loboda A Ens W, She YM, Standing KG: Characterization of a novel potyvirus isolated from maize in Israel. Phytopathology 2000, 90(5):505-513.

8. Wu JY, Ding JQ, Du YX, Xu YB, Zhang XC: Genetic analysis and molecular mapping of two dominant complementary genes determining resistance to sugarcane mosaic virus in maize. Euphytica 2007 156(3):355-364.

9. Louie $R$, Knoke JK, Findley WR: Elite maize germplasm-reactions to maize-dwarf mosaic and maize chlorotic dwarf viruses. Crop Sci 1990, 30(6):1210-1215.

10. Jiang H, Bai Y, Zhao Y, Liu W, Jiang H, Bai YJ, Zhao YM, Liu WZ: Studies on evaluation of resistance of maize ( Zea mays) germplasms to strain $B$ of maize dwarf mosaic virus in northeastern China. J Maize Sci 2002, 10(1):84-87.

11. Melchinger AE, Kuntze L, Gumber RK, Lubberstedt T, Fuchs E: Genetic basis of resistance to sugarcane mosaic virus in European maize germplasm. Theor App/ Genet 1998, 96(8):1151-1161.

12. Xia XC, Melchinger $A E$, Kuntze $L$, Lubberstedt T: Quantitative trait loci mapping of resistance to sugarcane mosaic virus in maize. Phytopathology 1999, 89(8):660-667.

13. Xu ML, Melchinger AE, Xia XC, Lubberstedt T: High-resolution mapping of loci conferring resistance to sugarcane mosaic virus in maize using RFLP, SSR, and AFLP markers. Mol Gen Genet 1999, 261(3):574-581.

14. Zhang SH, Li XH, Wang ZH, George ML, Jeffers D, Wang FG, Liu XD, Li MS, Yuan LX: QTL mapping for resistance to SCMV in chinese maize germplasm. Maydica 2003, 48(4):307-312.

15. Prazeres De Souza IR, Schuelter AR, Guimaraes $C T$, Schuster I, De Oliveira E, Redinbaugh M: Mapping QTL contributing to SCMV resistance in tropical maize. Hereditas 2008, 145(4):167-173.

16. Liu XH, Tan ZB, Rong TZ: Molecular mapping of a major QTL conferring resistance to SCMV based on immortal RIL population in maize. Euphytica 2009, 167(2):229-235

17. Ingvardsen CR, Xing YZ, Frei UK, Luebberstedt $T$ : Genetic and physical fine mapping of Scmv2, a potyvirus resistance gene in maize. Theor Appl Genet 2010, 120(8):1621-1634.

18. Dussle CM, Quint M, Melchinger AE, Xu ML, Lubberstedt T: Saturation of two chromosome regions conferring resistance to SCMV with SSR and AFLP markers by targeted BSA. Theor Appl Genet 2003, 106(3):485-493.

19. Yuan LX, Dussle CM, Melchinger AE, Utz HF, Lubberstedt T: Clustering of QTL conferring SCMV resistance in maize. Maydica 2003, 48(1):55-62.

20. Wisser RJ, Balint-Kurti PJ, Nelson RJ: The genetic architecture of disease resistance in maize: a synthesis of published studies.

Phytopathology 2006, 96(2):120-129.

21. Dussle CM, Melchinger AE, Kuntze L, Stork A, Lubberstedt T: Molecular mapping and gene action of $\mathrm{Scm} 1$ and $\mathrm{Scm} 2$, two major QTL contributing to SCMV resistance in maize. Plant Breed 2000, 119(4):299-303.

22. Yuan LX, Dussle CM, Muminovic J, Melchinger AE, Lubberstedt T: Targeted BSA mapping of Scmv1 and Scmv2 conferring resistance to SCMV using Pstl/Msel compared with EcoRI/Msel AFLP markers. Plant Breed 2004 123(5):434-437.

23. Springer NM, Ying $K$, Fu $Y$, Ji TM, Yeh $C T$, Jia $Y$, Wu W, Richmond $T$, Kitzman J, Rosenbaum $\mathrm{H}$, et al: Maize inbreds exhibit high levels of copy number variation (CNV) and presence/absence variation (PAV) in genome content. Plos Genet 2009, 5(11):e1000734.

24. Montes JM, Melchinger AE, Reif JC: Novel throughput phenotyping platforms in plant genetic studies. Trends Plant Sci 2007, 12(10):433-436.

25. Myles S, Peiffer J, Brown PJ, Ersoz ES, Zhang ZW, Costich DE, Buckler ES: Association mapping: critical considerations shift from genotyping to experimental design. Plant Cell 2009, 21(8):2194-2202.

26. Rafalski JA: Association genetics in crop improvement. Curr Opin Plant Biol 2010, 13(2):174-180.

27. Yan JB, Warburton M, Crouch J: Association mapping for enhancing maize (Zea mays L.) genetic improvement. Crop Sci 2011, 51(2):433-449.

28. Xing $Y Z$, Ingvardsen C, Salomon R, Lubberstedt T: Analysis of sugarcane mosaic virus resistance in maize in an isogenic dihybrid crossing scheme and implications for breeding potyvirus-resistant maize hybrids. Genome 2006, 49(10):1274-1282.

29. Salvi S, Tuberosa R: To clone or not to clone plant QTLs: present and future challenges. Trends Plant Sci 2005, 10(6):297-304.

30. Lai JS, Li RQ, Xu X, Jin WW, Xu ML, Zhao HN, Xiang ZK, Song WB, Ying $K$, Zhang M, Jiao YP, Ni PX, Zhang JG, Li D, Guo XS, Ye KX, Jian M, Wang B,
Zheng HS, Liang HQ, Zhang XQ, Wang SC, Chen SJ, Li JS, Fu Y: Genome-wide patterns of genetic variation among elite maize inbred lines. Nat Genet 2010, 42(11):1027-U1158.

31. Babiychuk E, Bouvier-Nave P, Compagnon V, Suzuki M, Muranaka T, Van Montagu M, Kushnir S, Schaller H: Allelic mutant series reveal distinct functions for arabidopsis cycloartenol synthase 1 in cell viability and plastid biogenesis. P Natl Acad Sci USA 2008, 105(8):3163-3168.

32. Griebel T, Zeier J: A role for beta-sitosterol to stigmasterol conversion in plant-pathogen interactions. Plant J 2010, 63(2):254-268.

33. Sindhu A, Chintamanani S, Brandt AS, Zanis M, Scofield SR, Johal GS: A guardian of grasses: specific origin and conservation of a unique disease-resistance gene in the grass lineage. P Natl Acad Sci USA 2008, 105(5):1762-1767.

34. Chintamanani S, Multani DS, Ruess H, Johal GS: Distinct mechanisms govern the dosage-dependent and developmentally regulated resistance conferred by the maize $\mathrm{Hm} 2$ gene. Mol Plant Microbe In 2008 , 21(1):79-86.

35. Tada Y, Spoel SH, Pajerowska-Mukhtar K, Mou ZL, Song JQ, Wang C, Zuo JR, Dong XN: Plant immunity requires conformational charges of NPR1 via S-nitrosylation and thioredoxins. Science 2008, 321(5891):952-956.

36. Sun LJ, Ren HY, Liu RX, Li BY, Wu TQ, Sun F, Liu HM, Wang XM, Dong HS: An h-Type thioredoxin functions in tobacco defense responses to two species of viruses and an abiotic oxidative stress. Mol Plant Microbe In 2010, 23(11):1470-1485.

37. Sweat TA, Wolpert TJ: Thioredoxin $h 5$ is required for victorin sensitivity mediated by a CC-NBS-LRR gene in arabidopsis. Plant Cell 2007, 19(2):673-687.

38. Lu YL, Zhang SH, Shah T, Xie CX, Hao ZF, Li XH, Farkhari M, Ribaut JM, Cao M, Rong TZ, Xu YB: Joint linkage-linkage disequilibrium mapping is a powerful approach to detecting quantitative trait loci underlying drought tolerance in maize. P Natl Acad Sci USA 2010, 107(45):19585-19590.

39. Tian F, Bradbury PJ, Brown PJ, Hung H, Sun Q, Flint-Garcia S, Rocheford TR, McMullen MD, Holland JB, Buckler ES: Genome-wide association study of leaf architecture in the maize nested association mapping population. Nat Genet 2011, 43(2):159-U113.

40. Li L, Li H, Li Q, Yang XH, Zheng DB, Warburton M, Chai YC, Zhang P, Guo YQ, Yan JB, Li JS: An 11-bp insertion in Zea mays fatb reduces the palmitic acid content of fatty acids in maize grain. Plos One 2011, 6(9):e24699.

41. Wisser RJ, Sun Q, Hulbert SH, Kresovich S, Nelson RJ: Identification and characterization of regions of the rice genome associated with broad-spectrum, quantitative disease resistance. Genetics 2005, 169(4):2277-2293.

42. Krattinger SG, Lagudah ES, Spielmeyer W, Singh RP, Huerta-Espino J, McFadden H, Bossolini E, Selter LL, Keller B: A putative ABC transporter confers durable resistance to multiple fungal pathogens in wheat. Science 2009, 323(5919):1360-1363.

43. Kump KL, Bradbury PJ, Wisser RJ, Buckler ES, Belcher AR, Oropeza-Rosas MA, Zwonitzer JC, Kresovich S, McMullen MD, Ware D, et al: Genome-wide association study of quantitative resistance to southern leaf blight in the maize nested association mapping population. Nat Genet 2011, 43(2):163-U120.

44. McMullen MD, Jones MW, Simcox KD, Louie R: 3 Genetic-loci contorl resistance to wheat streak mosaic -virus in the maize inbred PA405. Mol Plant Microbe In 1994, 7(6):708-712.

45. Jones MW, Redinbaugh MG, Louie R: The Mdm 1 locus and maize resistance to maize dwarf mosaic virus. Plant Dis 2007, 91(2):185-190.

46. Kou YJ, Wang SP: Broad-spectrum and durability: understanding of quantitative disease resistance. Curr Opin Plant Biol 2010, 13(2):181-185.

47. Murray MG, Thompson WF: Rapid isolation of high molecular-weight plant DNA. Nucleic Acids Res 1980, 8(19):4321-4325.

48. Yang Q, Yin GM, Guo YL, Zhang DF, Chen SJ, Xu ML: A major QTL for resistance to gibberella stalk rot in maize. Theor App/ Genet 2010, 121(4):673-687.

49. Li Q, Wan JM: SSRHunter: development of a local searching software for SSR sites. hereditas 2005, 27(5):808-810.

50. Edgar RC: MUSCLE: multiple sequence alignment with high accuracy and high throughput. Nucleic Acids Res 2004, 32(5):1792-1797.

51. Pritchard JK, Stephens M, Donnelly P: Inference of population structure using multilocus genotype data. Genetics 2000, 155(2):945-959. 
52. Falush D, Stephens M, Pritchard JK: Inference of population structure using multilocus genotype data: linked loci and correlated allele frequencies. Genetics 2003, 164(4):1567-1587.

53. Bradbury PJ, Zhang Z, Kroon DE, Casstevens TM, Ramdoss Y, Buckler ES: TASSEL: software for association mapping of complex traits in diverse samples. Bioinformatics 2007, 23(19):2633-2635.

doi:10.1186/1471-2229-13-162

Cite this article as: Tao et al:: Combined linkage and association mapping reveals candidates for Scmv1, a major locus involved in resistance to sugarcane mosaic virus (SCMV) in maize. BMC Plant Biology 2013 13:162.

\section{Submit your next manuscript to BioMed Central and take full advantage of:}

- Convenient online submission

- Thorough peer review

- No space constraints or color figure charges

- Immediate publication on acceptance

- Inclusion in PubMed, CAS, Scopus and Google Scholar

- Research which is freely available for redistribution 